\title{
Angular dependence of the secondary electron emission crystal current: Effects of surface modification
}

\author{
F. Peeters, E. R. Puckrin, a) and A. J. Slavin ${ }^{\text {a) }}$ \\ Department of Physics, Trent University, Peterborough, Ontario K9J 7B8, Canada
}

(Received 13 July 1989; accepted 28 October 1989)

\begin{abstract}
The variation of the angle between the incident electron beam and sample surface contributes in two ways to the secondary electron emission from single crystals. These contributions are a monotonically varying background due to the changing number of secondaries produced near the surface, and an oscillatory component previously explained as a bulk effect related to Kikuchi patterns. This work shows that for emission from a Au(111) surface, the background can be described well by a simple model based on the semiempirical theory of Kanaya and Kawakatsu. It also provides additional support for the explanation of the oscillations as a bulk effect by showing that the oscillation amplitude remains essentially unchanged either by surface sputtering or by the deposition of a thin lead layer. The presence of these oscillations requires some care in the use of the secondary electron crystal current as a measure of the thickness of thin metal films.
\end{abstract}

\section{INTRODUCTION}

When a metal crystal is exposed to a primary electron beam of energy between about 200 and $2000 \mathrm{eV}$, the secondary electron yield is greater than unity. ${ }^{1}$ This results in an effective positive current from the sample to ground, which has been called the secondary electron emission crystal current (SEECC). The variation of the SEECC as a function of the angle $\theta$ between the incident beam and the surface normal is shown for a cleaned and annealed $\mathrm{Au}(111)$ surface in Fig. 1 (a). For primary energies above $1000 \mathrm{eV}$, this variation has been interpreted as due to a combination of two contributions: (i) a monotonically varying background resulting from enhanced production of secondary electrons closer to the surface for increasing angles $\theta$ and (ii) an oscillatory structure caused by inverse Kikuchi effects (or inverse channeling of electrons) which cause the SEECC to peak at angles for which the incident beam is parallel to several low index planes. ${ }^{2-5}$ Thus, the oscillations are predicted to result primarily from the bulk structure of the crystal. The present work supports this conclusion by showing that the oscillation amplitude remains essentially unchanged either by sputtering or by the deposition of a thin $\mathrm{Pb}$ overlayer. It also shows that the background can be fitted well by a simple model based on the semiempirical theory of Kanaya and Kawakatsu ${ }^{6}$ for secondary electron emission.

The magnitude of the SEECC changes during metal film deposition by evaporation. A plot of the SEECC as a function of the deposition time shows a sharp break in slope at the completion of the first monolayer (ML).$^{7-10}$ It has been suggested that these changes can be used to determine film thickness once an initial calibration has been made. ${ }^{7}$ It will be shown that the rapid change in SEECC with angle indicates that considerable care is necessary in employing this technique.

\section{EXPERIMENTAL}

All experiments were carried out in an ultrahigh vacuum system with a base pressure below $1 \times 10^{-9}$ Torr. The single crystal gold sample ( $99.999 \%$ pure) was cut parallel to the
(111) plane $\left( \pm 1^{\circ}\right)$. The polycrystalline sample' was cut from Au foil (Alpha Products; $99.99 \%$ pure). The samples were mounted on a manipulator that allowed rotation about an axis perpendicular to the primary electron beam axis. For the $\mathrm{Au}(111)$ sample, the axis of rotation corresponded to the [211] axis within $2^{\circ}$ as determined by low-energy electron diffraction (LEED). Both gold samples were cleaned by argon ion bombardment at $900 \mathrm{~K}$. Following this cleaning procedure, no contamination could be detected with $\mathrm{Au}$ ger electron spectroscopy (AES). The electron gun (Perkin-Elmer Instruments model B78007) was operated to produce a beam current of $15.0 \pm 0.1 \mu \mathrm{A}$ at a primary energy $E_{P}$, of $1500.0 \pm 0.5 \mathrm{eV}$. The beam current was measured as the current from the sample to ground, with the sample biased at $+90 \mathrm{~V}$ relative to ground potential, and with the sample surface normal to the primary electron beam. The SEECC was also measured as the current from sample to ground, but with no applied voltage on the sample to allow the (low energy) secondary electrons to escape. The internal resistance of the ammeter was $1.25 \mathrm{k} \Omega$ for these measurements, which put a negligible bias of about $25 \mathrm{mV}$ on the sample.

\section{RESULTS AND DISCUSSION}

\section{A. Annealed surface}

Curve (a) in Fig. 1 for the cleaned and annealed Au(111) surface shows oscillations with maxima where expected for the incident beam parallel to the atomic planes given in parentheses. This curve is approximately symmetric about $0^{\circ}$ as required for this geometry. Note that such symmetry can be used to orient the crystal in the chamber. Raising the sample temperature to $900 \mathrm{~K}$ resulted in a decrease in the intensity of the fine structure in agreement with the work by Taub et $a l^{3}$

\section{B. Slowly varying background}

Kanaya and Kawakatsu ${ }^{6}$ have developed an expression for the secondary electron current based on the ejection of 


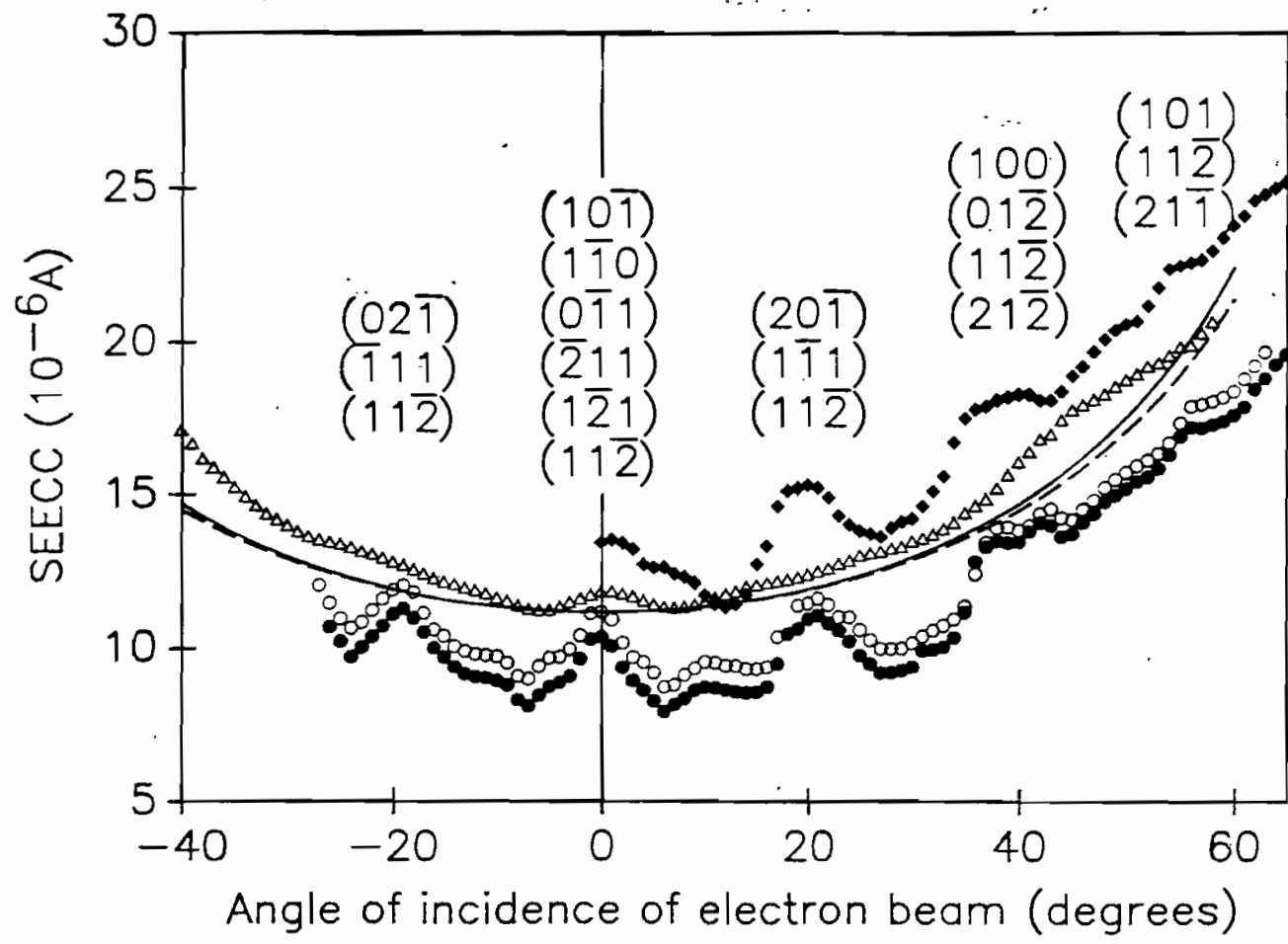

FIG. 1: The SEECC as a function of the angle between the direction of the incident electron beam and the sample normal. The numbers in parertheses are the indices of planes parallel to the incident electron beam. (a) (O) $\mathrm{Au}(111)$ surface annealed at $900 \mathrm{~K}$; (b) (-) theoretical curve neglecting backscattering and crystal structure; (c) (- - ) as (b) but with backscattering included; (d) (-) Au(111) surface sputtered for $15 \mathrm{~min}$; (e) $(\triangle$ ) polycrystalline Au sputtered, and annealed at $900 \mathrm{~K}$; (f) (\$) $\mathrm{Au}(111)$ surface covered by one $\mathrm{ML} \mathrm{Pb}$. core electrons from the solid. Although this is an oversimplification because it ignores the major loss mechanisms of plasmon and electron-hole creation (see, e.g., Ref. 11), it does provide a convenient parameterization in terms of measurable quantities, which they have tabulated for most elements. Specifically, they have shown that for nickel and lithium the dependence on incident angle of the slowly varying part of the SEECC is fitted acceptably (ignoring backscattering of electrons) by an expression of form

$\operatorname{SEECC} \propto \int_{0}^{1} \int_{0}^{1} \exp [-A y \cos \theta / z](1-y)^{1 / n-1} d z d y$,

where $A=K E_{p}^{\prime \prime}$ (experimental values of $K$ and $n$ are tabulated in Ref. 6 ), $z=\cos \phi$ ( $\phi$ is the angle of emission), and $y$ is a dimensionless parameter proportional to the depth in the sample at which a secondary electron is produced. Equation (1) is plotted in Fig. 1(b) using $n=4 / 3$ and $A=4.196$ for gold with $E_{p}=1500 \mathrm{eV}$. Including backscattering effects, Eq. (11) of Ref. 6 with a backscattering coefficient of $0.47^{6}$, results in the curve of Fig. 1(c). Both Figs. 1(b) and 1(c) have been normalized to the experimental value in Fig. 1(a) at $0^{\circ}$, since according to the theory of Taub and Stern, ${ }^{2}$ the increase in absorption of incident electrons moving parallel to low-index planes should not significantly affect the SEECC whereas the decrease in absorption at other angles will have a major effect. This argument is supported by the coincidence of curve (c) with most of the other maxima of curve (a).

\section{Effect of surface sputtering on the oscillations}

The SEECC was measured again following sputtering of the surface for $30 \mathrm{~min}$. This resulted in a reduced SEECC but dia not affect the intensity of the fine structure [Fig. 1(d)].
This can be explained as follows. Sputtering only destroys the crystallinity of the surface layer. Since electrons with energies of $1500 \mathrm{eV}$ penetrate several atomic layers into the sample (the inelastic mean free path is approximately ten $\mathrm{ML}^{12}$ ), inverse channeling will still occur below the surface where the crystal structure is not disturbed. Consequently, sputtering has little effect on the intensity of the oscillatory structure of the SEECC. The small, and approximately constant, decrease in the SEECC for the sputtered surface at all angles of incidence is probably caused by an increase in scattering and energy loss of the secondary electrons. This will reduce the secondary electron emission, independent of the angle of the incident electron beam. Annealing allows recrystallization of the sample surface, which returns the SEECC to its original (unsputtered) value.

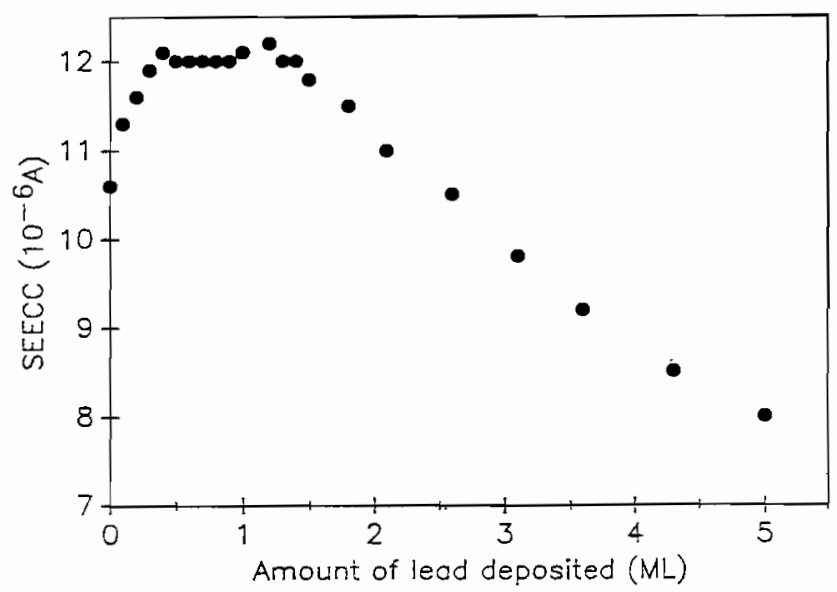

FIG. 2. The SEECC from Au(111) as a function of lead coverage. 
An additional point of interest is that in surface experiments, the absence of a LEED pattern may often je due to either surface damage or lack of bulk crystallinity. Measurement of the SEECC during sample rotation is an easy method to distinguish between these possibilities, since oscillations due to bulk crystallinity can be apparent even when surface damage is present.

\section{Polycrystalline sample}

For the polycrystalline sample, the SEECC [Fig. 1(e)] follows the theoretical background curve [Fig. 1(c)] fairly closely, except for a weak peak near $0^{\circ}$ and a weak broad peak near $45^{\circ}$. Polycrystalline Au foil annealed for several minutes has been reported to develop crystallites with predominantly (111) orientation. ${ }^{13}$ The weak peak at $0^{\circ}$ probably results from such crystallization. The origin of the broad peak near $45^{\circ}$ is unknown.

\section{E. Effect of a monolayer of lead}

The SEECC was also measured as a function of the thickness of $\mathrm{Pb}$ deposited on the $\mathrm{Au}(111)$ surface with the electron beam at normal incidence (Fig. 2). The SEECC increased relative to the value for clean $\mathrm{Au}(111)$ until the surface was covered by about $0.4 \pm 0.1 \mathrm{ML} \mathrm{Pb}$ as monitored by Auger electron spectroscopy. ${ }^{14}$ Then, the SEECC remained approximately constant until a coverage of about 1 $\mathrm{ML} \mathrm{Pb}$ was achieved. For larger $\mathrm{Pb}$ deposits, the SEECC began to decrease. This behavior confirms what was reported earlier for the same system. ${ }^{7}$

The dependence of the SEECC on the angle of the incident beam at a coverage of $1 \mathrm{ML} \mathrm{Pb}$ [Fig. 1(f)] was similar to that of Fig. 1(a), for the cleaned and annealed Au(111) surface. However, the SEECC was increased on average by about $3 \mu \mathrm{A}$ for all angles of incidence.

Argile et al. ${ }^{9}$ have suggested that the initial increase of the SEECC with the thickness of $\mathrm{Pb}$ deposits on $\mathrm{Cu}(111)$ is caused by a decrease in the work function of the sample. If this is also the case for the $\mathrm{Pb}-\mathrm{Au}$ system, then the increase in the SEECC should be constant for all directions of the incident electron beam, with little change in the amplitude of the fine structure. A comparison of Figs. 1(a) and 1(f) supports this explanation.

It has been proposed ${ }^{7}$ to use the break at a one ML coverage in the curve of SEECC versus deposition time to calibrate submonolayer coverages in terms of the SEECC. This technique depends on very small changes in the SEECC; for example, the SEECC from clean $\mathrm{Au}(111)$ and from one $\mathrm{ML}$ $\mathrm{Pb}$ on $\mathrm{Au}(111)$ differ only by about $10 \%$. Since a rotation of the sample by $5^{\circ}$ can account for a similar change in the SEECC, one must be careful to always return the sample to exactly the same position and orientation.

\section{CONCLUSION}

Measurements have been made of the SEECC from $\mathrm{Au}$ (111) and polycrystalline Au under various surface conditions. The results show that the variation of the background component of the SEECC with angle of incidence can be fitted well by the theory of Kanaya and Kawakatsu. It also provides additional support for Taub and Stern's explanation of the oscillations in the SEECC as a function of incident angle. Finally, we have pointed out the need to be aware of the strong angular dependence of the SEECC when using it as a measure of film thickness.

\section{ACKNOWLEDGMENTS}

The gold single crystal was generously supplied by Dr. M. Swanson of the University of North Carolina. This research has been supported by NSERC, Canada. E. Puckrin acknowledges the financial support of an NSERC Scholarship.

- Aiso at the Department of Physics, Queen's University, Kingston, Ontario K7L 3N6, Canada.

${ }^{1}$ A. J. Dekker, Solid State Physics (Prentice-Hall, New York, 1957), Chap. 17.

${ }^{2}$ R. M. Stern and H. Taub, Phys. Rev. Lett. 20, 1340 (1968).

${ }^{3}$ H. Taub, R. M. Stern, and V. F. Dvoryankin, Phys. Status Solidi 33, 573 (1969).

${ }^{4}$ R. M. Stern and H. Taub, CRC Crit. Rev. Solid State Sci. 1, 221 (1970).

${ }^{5}$ G. R. Brooker, A. M. B. Shaw, M. J. Whelan, and P. B. Hirsch, Philos. Mag. 16, 1185 (1967).

${ }^{6} \mathrm{~K}$. Kanaya and H. Kawakatsu, J. Phys D. 5, 1727 (1972).

${ }^{7}$ M. G. Barthès-Labrousse and G. E. Rhead, Surf. Sci. 116, 217 (1982) .

${ }^{8}$ S. D. Parker and G. E. Rhead, Surf. Sci. 167, 271 (1986).

${ }^{9} \mathrm{C}$. Argile, M. G. Barthès-Labrousse, and G. E. Rhead, Surf. Sci. 138, 181 (1984).

${ }^{10}$ S. D. Parker and P. J. Dobson, Surf. Sci. 171, 267 (1986).

${ }^{11}$ J. C. Ashley, C. J. Tung, and R. H. Ritchie, Surf. Sci. 81, 409 (1979)

${ }^{12}$ M. P. Seah and W. A. Dench, Surf. Interface Anal. 1, 2 (1979).

${ }^{13}$ J. G. MacMillan-Jones, F. A. Londry, and A. J. Slavin, Surf. Sci. 186, 357 (1987).

${ }^{14}$ F. Peeters and A. J. Slavin, Surf. Sci. 214, 85 (1989). 